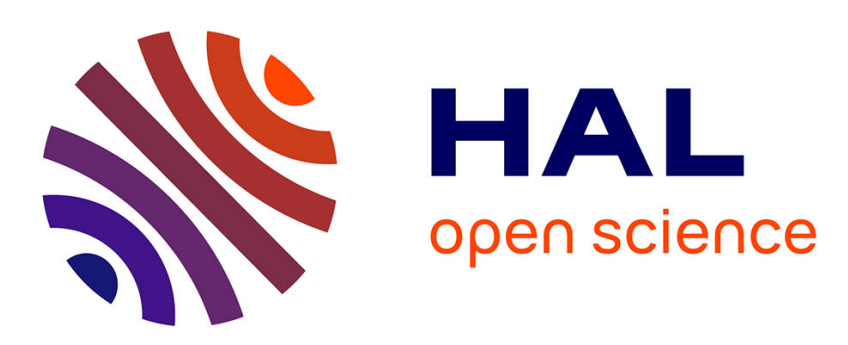

\title{
Optimizing Composites Forming Processes by Applying the Proper Generalized Decomposition
}

Chady Ghnatios, Francisco Chinesta, Elías Cueto, Adrien Leygue, Arnaud Poitou

\section{- To cite this version:}

Chady Ghnatios, Francisco Chinesta, Elías Cueto, Adrien Leygue, Arnaud Poitou. Optimizing Composites Forming Processes by Applying the Proper Generalized Decomposition. Advances in Material Processing Technologies (AMPT 2010), 2010, Paris, France. pp.197-202, 10.1063/1.3552440 . hal01008639

\section{HAL Id: hal-01008639 \\ https://hal.science/hal-01008639}

Submitted on 5 Nov 2017

HAL is a multi-disciplinary open access archive for the deposit and dissemination of scientific research documents, whether they are published or not. The documents may come from teaching and research institutions in France or abroad, or from public or private research centers.
L'archive ouverte pluridisciplinaire HAL, est destinée au dépôt et à la diffusion de documents scientifiques de niveau recherche, publiés ou non, émanant des établissements d'enseignement et de recherche français ou étrangers, des laboratoires publics ou privés. 


\title{
Optimizing Composites Forming Processes by Applying the Proper Generalized Decomposition
}

\author{
C. Ghnatios*, F. Chinesta*, E. Cueto ${ }^{\dagger}$, A. Leygue ${ }^{* *}$ and A. Poitou** \\ ${ }^{*}$ EADS Corporate Foundation International Chair \\ GeM : Ecole centrale de Nantes, Université de Nantes \\ 1 rue de la Noe, BP 92101, F-44321 Nantes cedex 3, France \\ Chady.Ghnatios@ec-nantes.fr; Francisco.Chinesta@ec-nantes.fr \\ ${ }^{\dagger}$ Campus Rio Ebro. - Edificio Agustin de Betancourt \\ University of Zaragoza \\ Maria de Luna, 7, E-50018 Zaragoza, Spain \\ ecueto@unizar.es \\ ${ }^{*}$ GeM : Ecole Centrale de Nantes-Université de Nantes \\ 1 rue de la Noë, BP 92101, F-44321 Nantes cedex 3, France \\ Adrien.Leygue@ec-nantes.fr; Arnaud.Poitou@ec-nantes.fr
}

\begin{abstract}
Different computational methods are nowadays active research topics in computational mechanics. Different strategies have been proposed, the main challenge being always the computing cost induced by complex problems exhibiting multiple degrees of freedom. In this paper we present the Proper Generalized Decompositions or PGD, a way of addressing an efficient solution for multidimensional problems [1]. Moreover, we will apply this technique on the design of dies. The optimal choice of the design parameters, in our case the temperatures prescribed on different regions of the die wall, is a difficult task. In general some values are chosen for the thermal simulations performed. Then a cost function is evaluated and the temperatures are modified in order to reduce such function. Obviously, for each choice of the temperatures prescribed on the different regions of the die walls, a thermal problem must be solved. Thus, optimization becomes too expensive from the computation time point of view. The PGD allows solving only once a thermal model for any temperature prescribed on the different regions of the die wall. Optimizations as well as inverse identification become an easy matter.
\end{abstract}

Keywords: PGD, parametric model, model reduction, optimisation

\section{INTRODUCTION}

Despite the spectacular progress in the context of computational mechanics in the last few decades, the efficient treatment of multidimensional models needs further developments [2]. In fact, this type of problems can't be solved by a classical technique like the finite element method, because of the time cost involved. Moreover, nowadays the fine description of the thermodynamics and structure of materials introduces some specific challenges related to the impressive number of degrees of freedom required for solving problems, which generates such meshes of multidimensional models. Furthermore, usual models encountered in computational physics are solved for specific conditions, but can also be transformed to multidimensional models allowing the computation of a very general solution. For example, in the pultrusion process, we can solve the equation of heat for all possible boundary conditions. Thus, we can optimize the system to a 
global optimum. Solving this equation is now possible due to the new numerical method so called the "Proper General Decomposition" or PGD, as we will describe later. In fact, when using PGD, the complexity of the problem scales linearly with its dimension, enabling the circumvention of the curse of dimensionality [1]. This technique has been successfully applied in a variety of linear, non linear, stationary and transient problems [3] [4].

In this paper, we will show the interest of multidimensional models by solving problems with parametric boundary conditions. Obviously, usual finite element procedures, for each choice of the boundary condition prescribed on a region of the boundary, a thermal problem must be solved. Thus, optimization becomes too expensive from the computation time point of view. The PGD allows solving only once a general model for any boundary condition prescribed on any chosen region of the mesh. Optimization as well as inverse identification becomes an easy matter.

\section{PARAMETRIC BOUNDARY CONDITIONS}

Often optimizing a process can be modelled as the optimal choice of a set of parameters, including sometimes some boundary conditions. This is, for example, the case of pultrusion, one of several manufacturing processes for polymer composites [5]. There is also too many other interests in computing parametric boundary conditions, which are currently being tested.

\section{Illustrating the construction of a solution using PGD}

In what follows, we show the construction of the solution, using PGD, of the following simple parametric heat transfer equation as an example:

$$
\frac{\partial u}{\partial t}-k \Delta u-f=0
$$

where $(\mathbf{x}, t, k) \in \Omega \times I \times \mathfrak{I}$, and for the sake of simplicity the source term is assumed to be constant, i.e. $f=$ cte. Because the conductivity is considered unknown, it is assumed as a new coordinate defined in the interval $\mathfrak{I}$. So, in this case, the solution of Eq. (1) is searched under the form:

$$
u(\mathbf{x}, t, k) \approx \sum_{i=1}^{i=N} X_{i}(\mathbf{x}) \cdot T_{i}(t) \cdot K_{i}(k)
$$

To start the algorithm, we assume that the approximation at iteration $n$ is already known. At present iteration we look for enrichment, thus $u$ writes:

$$
u^{n}(\mathbf{x}, t, k)=\sum_{i=1}^{i=n} X_{i}(\mathbf{x}) \cdot T_{i}(t) \cdot K_{i}(k)+R(\mathbf{x}) \cdot S(t) \cdot W(k)
$$

where $R(\mathbf{x}) \cdot S(t) \cdot W(k)$ are respectively $X_{n+1}(\mathbf{x}) \cdot T_{n+1}(t) \cdot K_{n+1}(k)$. Before solving 
the resulting non linear model related to the calculation of these three functions, a model linearization is performed. The simplest choice consists in using an alternating directions fixed point algorithm. First of all, we proceed by assuming $S(t)$ and $W(k)$ given at the previous iteration of the non-linear solver and then computing $R(\mathbf{x})$. From the just updated $R(\mathbf{x})$ and previously used $W(k)$ we can update $S(t)$. Finally from the just computed $R(\mathbf{x})$ and $S(t)$ we update $W(k)$. The procedure continues until reaching convergence. The converged functions $R(\mathbf{x}), S(t)$ and $W(k)$ allow defining all needed functions : $X_{n+1}(\mathbf{x})=R(\mathbf{x}), T_{n+1}(t)=S(t)$ and $K_{n+1}(k)=W(k)$ [1].

Remark 1. The construction of each term in the sum (2) needs a certain number of iterations because of the non linearity of the problem related with the approximation (3). Let $m=\sum_{i=1}^{i=N} m_{i}$ be the total number of iterations involved in the construction of the separated approximation (2), which rarely exceeds ten. On the other hand the number of sums $N$ needed to approximate the solution of a given problem depends on the solution regularity itself, but all the experiments carried out reveal that this number ranges from few tens to few hundreds. Thus, we can conclude that the complexity of the solution procedure is of some hundreds of $3 D$ solutions (the cost related to the one dimensional problems being negligible with respect to the $3 D$ problems [1]). Now, if we assume a classical approach, we should solve a $3 D$ problem at each time step and for each value of the parameter $k$. In [6] we proved that the CPU time savings by applying the PGD can be of several orders of magnitude.

Remark 2. This section shortly described is the most basic algorithm for constructing the sum (2). There are other ways to construct solutions using PGD, with faster convergence [7].

\section{Basic example using parametric boundary conditions}

To fixe the ideas, let's start by considering the basic one dimension differential equation that follows:

$$
-\frac{\partial^{2} T}{\partial x^{2}}=1
$$

Let's consider that we need to solve this equation for $x \in \Omega$ where $\Omega=[0 ; L]$ and for the following boundary conditions:

$$
\left\{\begin{array}{l}
T(0)=0 \\
T(L)=y
\end{array}\right.
$$

where $y$ is a variable parameter inside a domain, noted $\alpha$. Thus, in the classical methods, we will have to solve this equation for every single value of $y$. In the PGD method, we can include $y$ as an extra coordinate in the system, since this doesn't involve dramatically the complexity of the problem. Thus, we first write a function, $f(x, y)$ that satisfies all boundary conditions. For our example, we can use:

$$
f(x, y)=\frac{x \cdot y}{L}
$$


Since, as noted on the page before, $T$ can be written as a sum of $N$ products of functions $X(x)$ and $Y(y)$, and introducing the values of our boundary condition $f(x, y)$ as first mode of the solution, $T(x, y)$ writes now:

$$
T(x, y)=X_{1}(x) \times Y_{1}(y)+\sum_{i=2}^{i=N} X_{i}(x) . Y_{i}(y)
$$

where :

$$
X_{1}(x) \times Y_{1}(y)=\frac{x \cdot y}{L}
$$

Note that $X_{1}(x)$ and $Y_{1}(y)$ can be found using a singular value decomposition. Now we will go back to write the weak form of the problem, the trial function and test function write respectively as:

$$
\begin{gathered}
T(x, y)=X_{1}(x) Y_{1}(y)+\sum_{i=2}^{i=n} X_{i}(x) Y_{i}(y)+R(x) S(y) \\
T^{*}(x, y)=R^{*}(x) S(y)+R(x) S^{*}(y)
\end{gathered}
$$

- By replacing equations (9) and (10) into equation (4) and considering $S(y)$ known, so $S^{*}=0$ we have the first weak form to solve:

$$
\int_{\alpha} \int_{\Omega}\left(R Y_{1} \frac{\partial^{2} X_{1}}{\partial x^{2}}+R \sum_{i=2}^{i=n} \frac{\partial^{2} X_{i}}{\partial x^{2}} Y_{i}+\frac{\partial^{2} R}{\partial x^{2}} R S-R\right)=0
$$

- Now repeating the same procedure considering $R(x)$ known, thus $R^{*}=0$ and the second weak form:

$$
\int_{\Omega} \int_{\alpha}\left(S Y_{1} \frac{\partial^{2} X_{1}}{\partial x^{2}}+S \sum_{i=2}^{i=n} \frac{\partial^{2} X_{i}}{\partial x^{2}} Y_{i}+\frac{\partial^{2} R}{\partial x^{2}} S^{2}-S\right)=0
$$

Now, we should iterate equations (11) and (12) until convergence. Solutions are found for homogenous Dirichlet boundary conditions. This is a must, because our boundary conditions are already satisfied by first mode. The new modes will converge to the good solution taking in consideration the boundary conditions. Results of this problem are shown in figure 1.

\section{APPLYING PGD FOR OPTIMIZATION}

In this section, we will treat the problem of axisymmetric pultrusion. This is a continuous process to produce constant cross-sectional profile composites. For decades, engineers relied on experience to define optimal parameters for pultrusion process [8]. However, with the fast growing use of this technique [9], more effective methods must be used for the design of this process. Many numerical models have been presented, each with his own advantages, but most of the simulation procedures were designed for particular 


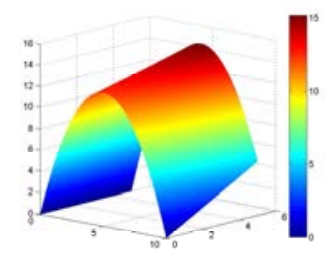

Figure 1. Solution of equation (4) for parametric boundary condition

cases [8]. In fact, each have chosen his own boundary conditions to solve a two dimensional heat transfer equation based on Fourier's law. This axisymmetric equation can be written as:

$$
\rho c\left(\frac{\partial T}{\partial t}+u \frac{\partial T}{\partial z}\right)=\frac{1}{r} \frac{\partial}{\partial r}\left(k_{r} \frac{\partial T}{\partial r}\right)+\frac{\partial}{\partial z}\left(k_{z} \frac{\partial T}{\partial z}\right)+q
$$

where $k_{r}$ and $k_{z}$ are the thermal conductivities in radial and axial directions respectively, $q$ is the internal heat generated by the resin curing reaction, $\rho$ is the density, $c$ is the specific heat and $u$ the pull speed. We will solve this problem in a die with three heating stages, each one is considered as a Dirichlet boundary condition. Obviously, each one will be considered as a parametric boundary condition, and a new coordinate. The pull speed $u$ is also a parameter of the problem, and a coordinate is assigned to it. Thus, the solution $T$ writes as :

$$
T\left(x, y, \theta_{1}, \theta_{2}, \theta_{3}, u\right)=\sum_{i=1}^{i=N} F_{i}(x, y) \times \Theta_{1 i}\left(\theta_{1}\right) \times \Theta_{2 i}\left(\theta_{2}\right) \times \Theta_{3 i}\left(\theta_{3}\right) \times U_{i}(u)
$$

while the boundary conditions write as :

$$
\left\{\begin{array}{l}
T\left(0, y, \theta_{1}, \theta_{2}, \theta_{3}, u\right)=T_{0} \\
T\left(x, y, \theta_{1}, \theta_{2}, \theta_{3}, u\right)=\theta_{1} \text { for } x \in\left[\varepsilon ; L_{1}-\varepsilon\right] \text { and }(y=0 \text { or } y=h) \\
T\left(x, y, \theta_{1}, \theta_{2}, \theta_{3}, u\right)=\theta_{2} \text { for } x \in\left[L_{1}+\varepsilon ; L_{2}-\varepsilon\right] \text { and }(y=0 \text { or } y=h) \\
T\left(x, y, \theta_{1}, \theta_{2}, \theta_{3}, u\right)=\theta_{3} \text { for } x \in\left[L_{2}+\varepsilon ; L_{3}-\varepsilon\right] \text { and }(y=0 \text { or } y=h) \\
\nabla T\left(L_{3}, y, \theta_{1}, \theta_{2}, \theta_{3}, u\right)=0
\end{array}\right.
$$

where $L_{1}$ and $L_{2}$ are the values of $x$ coordinate at the entrance of heater II and III respectively. $L_{3}$ is the value of the $x$ coordinate at the exit of the die. $h$ is the width of the die. To solve this problem, we will use a set of four initial modes to define (15) and use the same technique as described on the page before. Solution for one particular case is shown in figure 2.

We compared the solution represented in figure 2 to the finite element one for same boundary condition, and we have found a difference less than $10^{-5}$ for $N$ going up to 30 modes. 


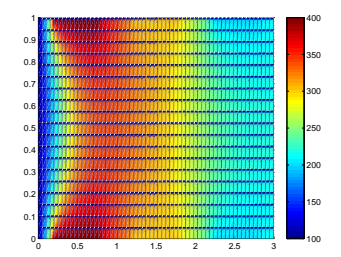

Figure 2. Solution for $U_{0}=100 \mathrm{~K}$, heater temperatures are respectively $400 \mathrm{~K}, 300 \mathrm{~K}$ and $200 \mathrm{~K}$ for heater I, II and III. Pull speed is $0.1 \mathrm{~m} / \mathrm{s}$

\section{CONCLUSION}

In this paper, we summarized a way to construct the PGD, and we proposed a new approach for solving problems involving parametric boundary conditions. This new view of problems promises very important changes in optimization. As we have seen, to many mechanical optimization problems consist of choosing a set of parameters. However, computing solution for all boundary conditions is not enough to optimize the process. Further treatment needs to be performed in a huge multidimensional space, where human imagination is limited. Some algorithms are currently being tested, trying to reach a global optimum of each process.

\section{REFERENCES}

1. F. Chinesta, A. Ammar, and E. Cueto, Archives of Computational Methods in Engineering (In Press).

2. F. Chinesta, A. Ammar, and E. Cueto, On the use of model reduction in material and processes simulation: A way for realizing simulation dreams, Tech. rep., EADS Corporate Foundation Chaire, GeM CNRS - Centrale Nantes (In press).

3. A. Ammar, B. Mokdad, F. Chinesta, and R. Keunings, J. Non Newtonian Fluid Mech. 139, 153-176 (2006).

4. A. Ammar, B. Mokdad, F. Chinesta, and R. Keunings, J. Non Newtonian Fluid Mech. 144, 98-121 (2007).

5. J. Li, S. C. Joshi, and Y. C. Lam, Composites Science and Technology 62 (3), 457-467 (2002).

6. F. Chinesta, A. Ammar, and E. Cueto, International Journal of Numerical Methods in Engineering (In press).

7. E. Pruliere, F. Chinesta, and A. Ammar, Mathematics And Computer Simualtion (In press).

8. J. Martin, Reinforced Plastics 50(5), 20-27 (2006).

9. A. Jacob, Reinforced Plastics 50(5), 38-41 (2006). 